\title{
The seroepidemiology of varicella in Italy
}

\author{
G. GABUTTI ${ }^{12 *}$, C. PENNA ${ }^{1}$, M. ROSSI ${ }^{1}$, S. SALMASO ${ }^{3}$, M. C. ROTA ${ }^{3}$, \\ A. BELLA ${ }^{3}$, P. CROVARI ${ }^{1}$ and The Serological Study Group $\dagger$ \\ ${ }^{1}$ Department of Health Sciences, Hygiene and Preventive Medicine Section, University of Genoa, Italy \\ ${ }^{2}$ Laboratory of Hygiene, Department of Biology, University of Lecce, Italy \\ ${ }^{3}$ Laboratory of Epidemiology and Biostatistics, Istituto Superiore di Sanità, Rome, Italy
}

(Accepted 10 December 2000)

\section{SUMMARY}

We conducted a seroepidemiological study to evaluate the potential impact of mass varicella vaccination on the transmission of varicella-zoster virus (VZV) in Italy, where vaccination is not mandatory. We tested 3179 serum samples, collected from September 1996 to October 1997, for specific anti-VZV antibodies using a commercially available ELISA. The results confirmed that varicella typically involves children (82.1\% seroprevalence among 10 - to 14 year-olds) and that the mean age of acquiring the infection seems to be increasing. The results also revealed that southern Italy, compared to the rest of the country, has a greater circulation of VZV and an earlier age of acquisition. The potential impact of mass vaccination among 12to 18-month-old children on the epidemiological trend of the infection must be carefully considered, in that failure to reach high levels of coverage could lead to an increase in the mean age of acquisition, which is already occurring in Italy.

\section{INTRODUCTION}

Varicella (chickenpox) is an acute, highly infectious disease caused by the alpha-herpes virus varicellazoster virus (VZV), which has a worldwide distribution and is typically associated with epidemics among children [1]. VZV is capable of persisting in a latent form in the spinal and cranial ganglia and reactivation can occur many years after the first infection, resulting in the onset of herpes zoster (shingles) [2].

* Author for correspondence: Laboratory of Hygiene, Department of Biology, University of Lecce, Via Provinciale Lecce-Monteroni, 73100 Lecce, Italy.

$\dagger$ Members of the Serological Study Group: S. Barbuti, M. C. Berlinghieri, W. Caraccio, E. Ciotoli, L. Cosentino, E. Del Prete, A. Favero, A. Focà, M. Fringuelli, C. Germinario, A. Giammanco, A. Goglio, S. Grandesso, M. Greco, F. Leoncini, G. Maistri, D. Marchetti, P. Martelli, P. Montanera, A. Mundo, G. Obino, S. Oliva, S. Pegoretti, V. Perani, P. Quaranta, G. Riario Sforza, G. Santini, L. Simula, L. Titone, and M. Tronci.
The postnatal form of varicella is usually acquired between the ages of 5 and 9 years and generally has a benign clinical course. However, the infection can lead to serious complications that are sometimes fatal, especially in immunocompromised individuals and in the first year of life [3]. Furthermore, complications occur more frequently in adults; hospitalization and length of stay reflect the age-specific severity of varicella. The hospitalization rate per varicella case is high in children under 2 years of age, becomes very low in 2- to 18-year-olds and then increases significantly throughout adult life $[4,5]$.

A neonatal form of varicella can occur if the mother acquires the infection in the first months of pregnancy $[6,7]$. If the infection is acquired 15 days or less prior to delivery, the newborn presents with typical rash in at least $25 \%$ of cases, whereas if the infection is acquired from 5 days before to 2 days after delivery, the clinical course in the newborn becomes par- 
ticularly serious, with up to $30 \%$ mortality, since the newborn is immunologically immature $[8,9]$.

However, since the incidence peaks among individuals between 5 and 9 years of age, complications are relatively rare and there is only a low probability that a woman will reach reproductive age and still be susceptible to VZV. In these geographic areas, varicella is endemic, with periodic peak years. Between peak years, there are frequent local or regional reports of varicella, with epidemic characteristics, within closed communities consisting of a high number of susceptible individuals (e.g. schools, military barracks, and hospitals). Moreover, it is known that approximately $15-25 \%$ of individuals will acquire herpes zoster in their lifetime, over $70 \%$ of which are in adults $[2,10]$.

By contrast, in tropical countries, the incidence of varicella peaks at older ages and the disease is thus associated with a greater rate of complications [11]. This delay in acquiring the infection could be due to the fact that a larger proportion of the population lives in rural, less densely populated areas; the delay could also be due to the interference of other circulating herpesviruses (HSV in particular) or to the aetiological agent being less resistant, in relation to specific environmental conditions [12, 13].

A live-attenuated varicella vaccine has been available for several decades [14]; it is safe and effective, mainly in preventing severe disease [15]. The first clinical trials on this vaccine were conducted among immunocompromised children and later among healthy children and adults [1]. The American Academy of Pediatrics currently recommends vaccinating all infants 12-18 months of age (or in any case performing vaccination before 13 years of age, if the individual is still susceptible) $[16,17]$. However, as with any mass paediatric vaccination, the consequences of the extensive use of varicella vaccine must be thoroughly evaluated from an epidemiological point of view. This entails evaluating how mass vaccination, especially if there are low coverage rates, can modify the transmission of the aetiological agent. To this end, we conducted a seroepidemiological study in Italy, where varicella vaccination is not mandatory.

\section{METHODS}

Although varicella vaccination is not mandatory in Italy, the Ministry of Health has actively recommended vaccinating anti-VZV-negative individuals who belong to risk categories (e.g. individuals affected by chronic renal failure and lymphoproliferative diseases, in particular leukaemia; and candidates for liver, bone-marrow, and kidney transplant) [18]. The notification of varicella in Italy is mandatory [19], and all notified cases are also recorded by the Italian National Institute for Statistics (ISTAT).

To determine the morbidity of varicella in Italy, we reviewed data from ISTAT on all cases reported in Italy's 20 geographical regions for the period 1961-96 (the most recent available data). To evaluate time trends in morbidity (per 100000 individuals), we reviewed data from censuses conducted in the years 1961, 1971, 1981, and 1991 (in Italy, national censuses are conducted every 10 years). The standardized rates were computed using the 1991 census population as reference population. Time trends were also evaluated for specific age-groups (i.e. 0-4, 5-9, 10-14, 15-24, $25-44$, and $\geqslant 45$ years), and we identified eventual changes in the percentage of varicella cases notified in the various age-groups in the period considered. Finally, for the period 1980-96, we studied the trend of varicella morbidity by geographic area (i.e. northern, central and southern Italy).

For the seroprevalence analysis, we collected anonymous unlinked samples of residual sera from routine laboratory testing, provided by a reference laboratory in each region. We excluded samples from individuals known to have an immunosuppressive or acute infectious disease and those from individuals who had recently undergone a blood transfusion. All individuals who provided serum samples gave verbal informed consent; consent for minors was provided by parents or legal guardians.

Based on a previous document [20], we calculated that a minimum of 3400 sera samples would be needed for the seroprevalence analysis. Specifically, we needed 100 samples for each 1-year interval in the age range 0-19 years (a total of 2000 samples) and 200 samples for each 5-year or 10-year interval for ages 20 years and over (i.e. 20-24, 25-29, 30-34, 35-39, 40-49, $50-59$, and 60 and over, for a total of 1400 samples). Eighteen of Italy's regions provided serum samples, which were divided by age-group (for samples from individuals less than 1 year of age, the age in months was specified). A total of 3569 samples were collected from September 1996 to October 1997 and were stored at $-20^{\circ} \mathrm{C}$ until tested for varicella antibodies.

Anti-VZV specific antibodies were detected using a commercially available ELISA (Enzygnost anti$\mathrm{VZV} / \mathrm{IgG}$, Dade Behring GmbH), which according to 


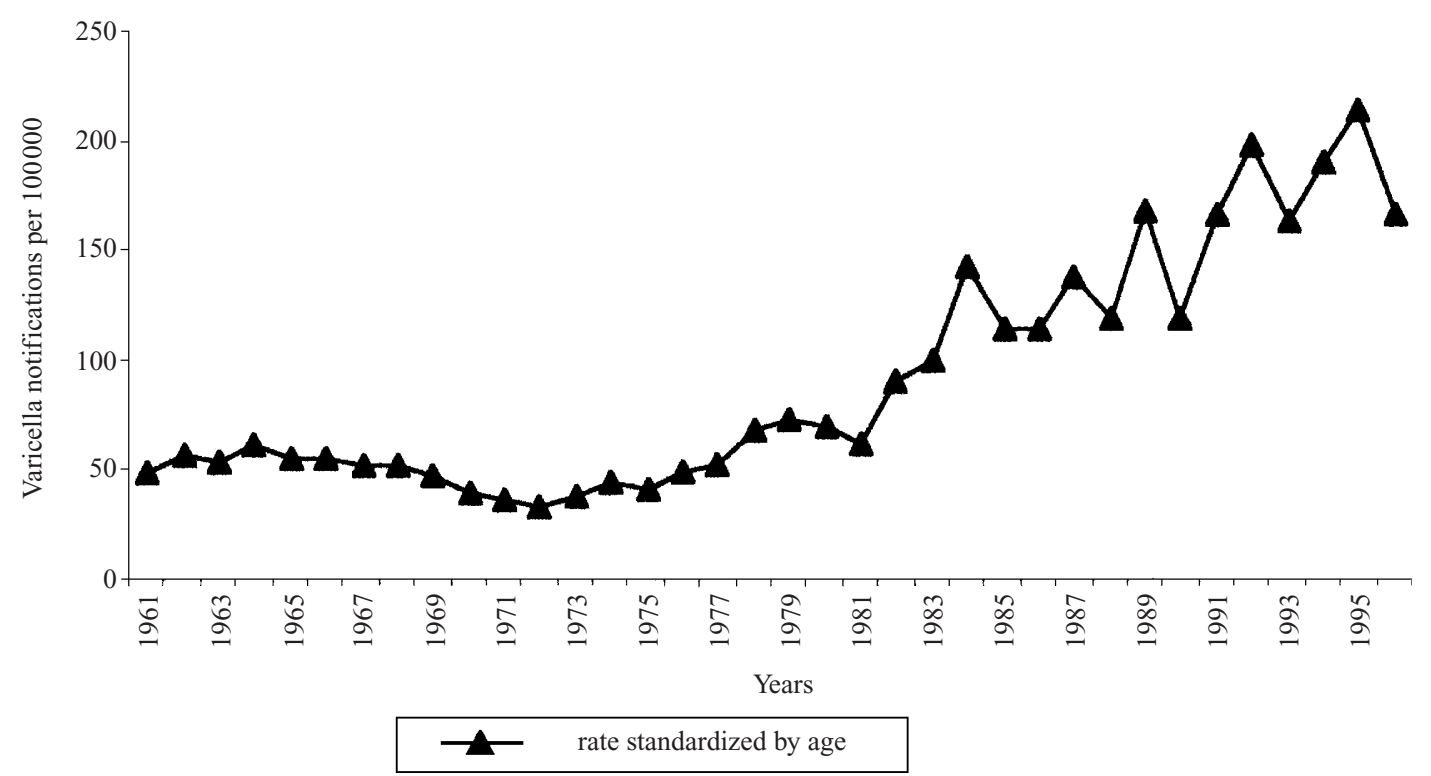

Fig. 1. Varicella morbidity in Italy 1961-96.

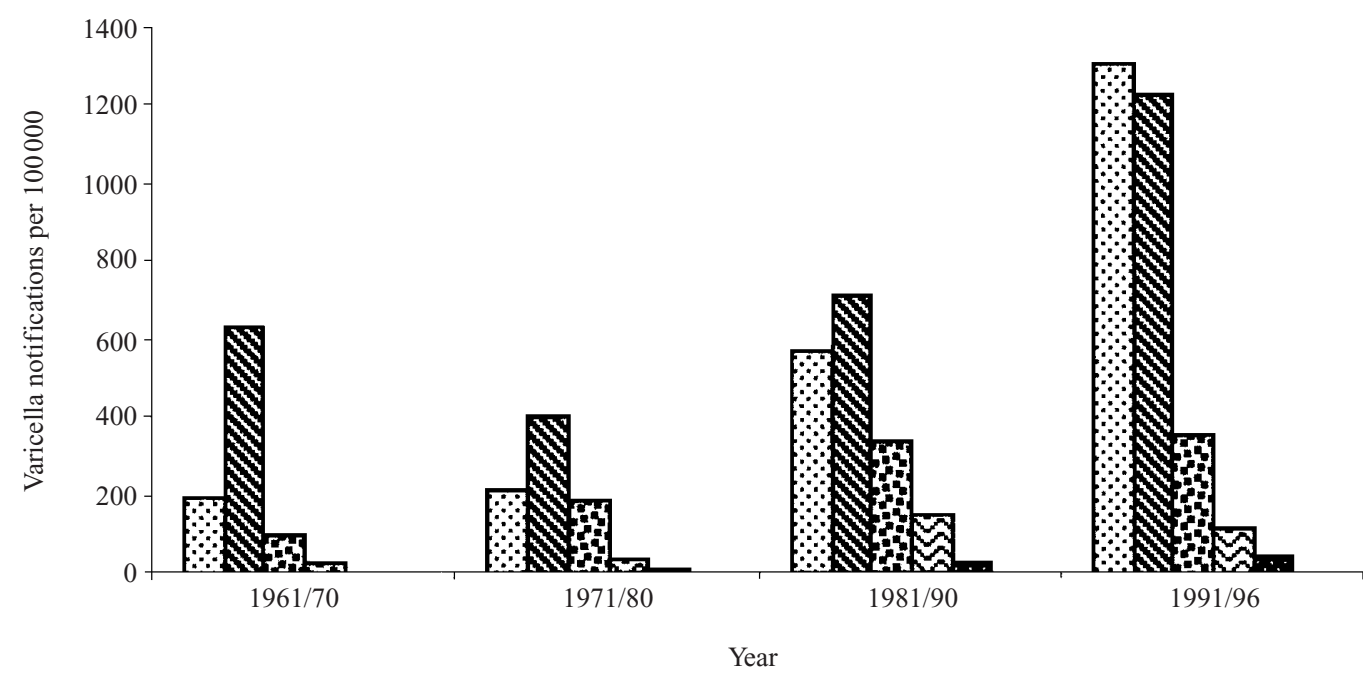

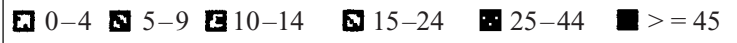

Fig. 2. Varicella morbidity by age-group in Italy 1961-96.

the manufacturer has a sensitivity of $99 \cdot 3 \%$ and a specificity of $100 \%$. Sera were classified as negative if the OD was less than $0 \cdot 100$ and as positive if higher than $0 \cdot 200$; sera with OD reproducibly between $0 \cdot 100$ and $0 \cdot 200$ were classified as borderline. The antibody status, expressed in $\mathrm{mIU} / \mathrm{ml}$, was based on the International Standard for Varicella-Zoster Immunoglobulin (50 IU) of the World Health Organization.

The statistical comparison of data was performed using the $\chi^{2}$ test (comparison among percentages) and the Student $t$ test (comparison among means). Geometric mean titres (GMT) were computed on all antibody titres as arithmetic means of logarithmically transformed values.

\section{RESULTS}

The trend of morbidity showed that in Italy varicella is endemic-epidemic (Fig. 1); from 1982 to 1996 there was a trend of increase in morbidity. The age-specific trend of morbidity, comparing data from the periods 1961-70, 1971-80, 1981-90 and 1991-6, showed significant changes $[0-4$ years $(P<0 \cdot 01)$; 5-9 years $(P<0 \cdot 01)$; 10-14 years $(P<0 \cdot 01) ; 15-24$ years 


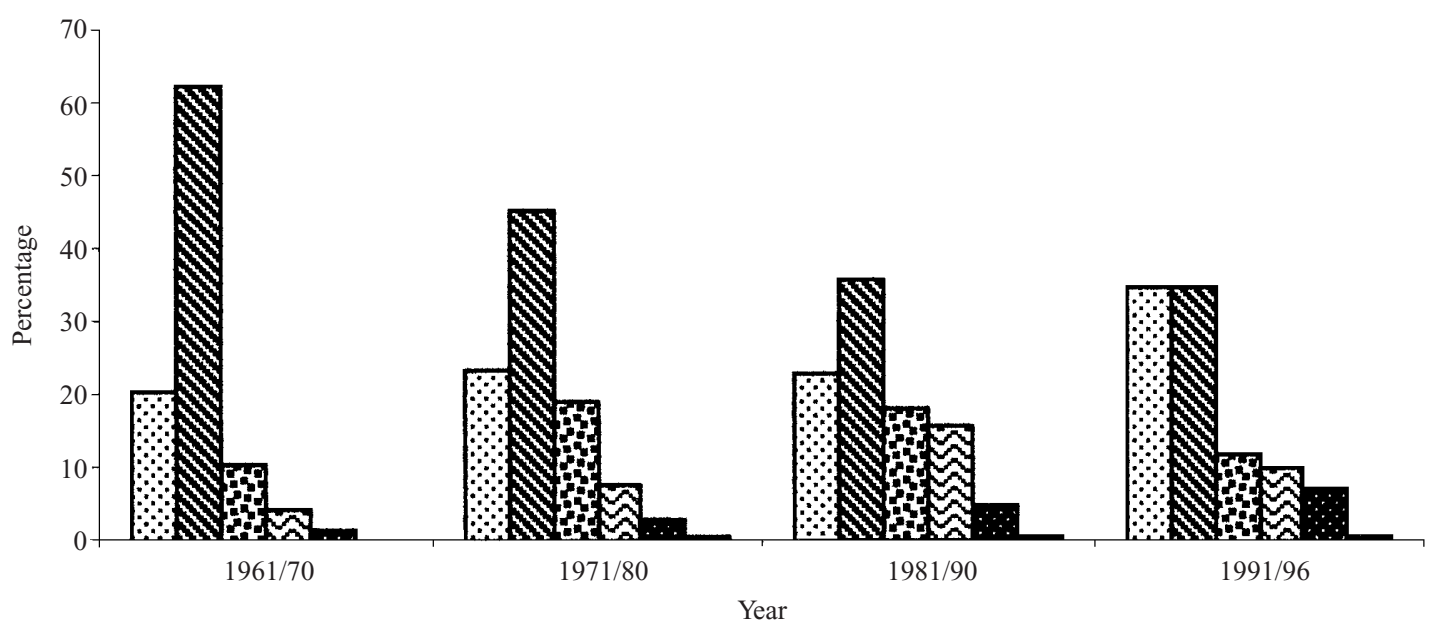

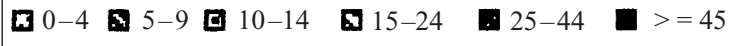

Fig. 3. Percentage of varicella notification by age-group in Italy 1961-96.

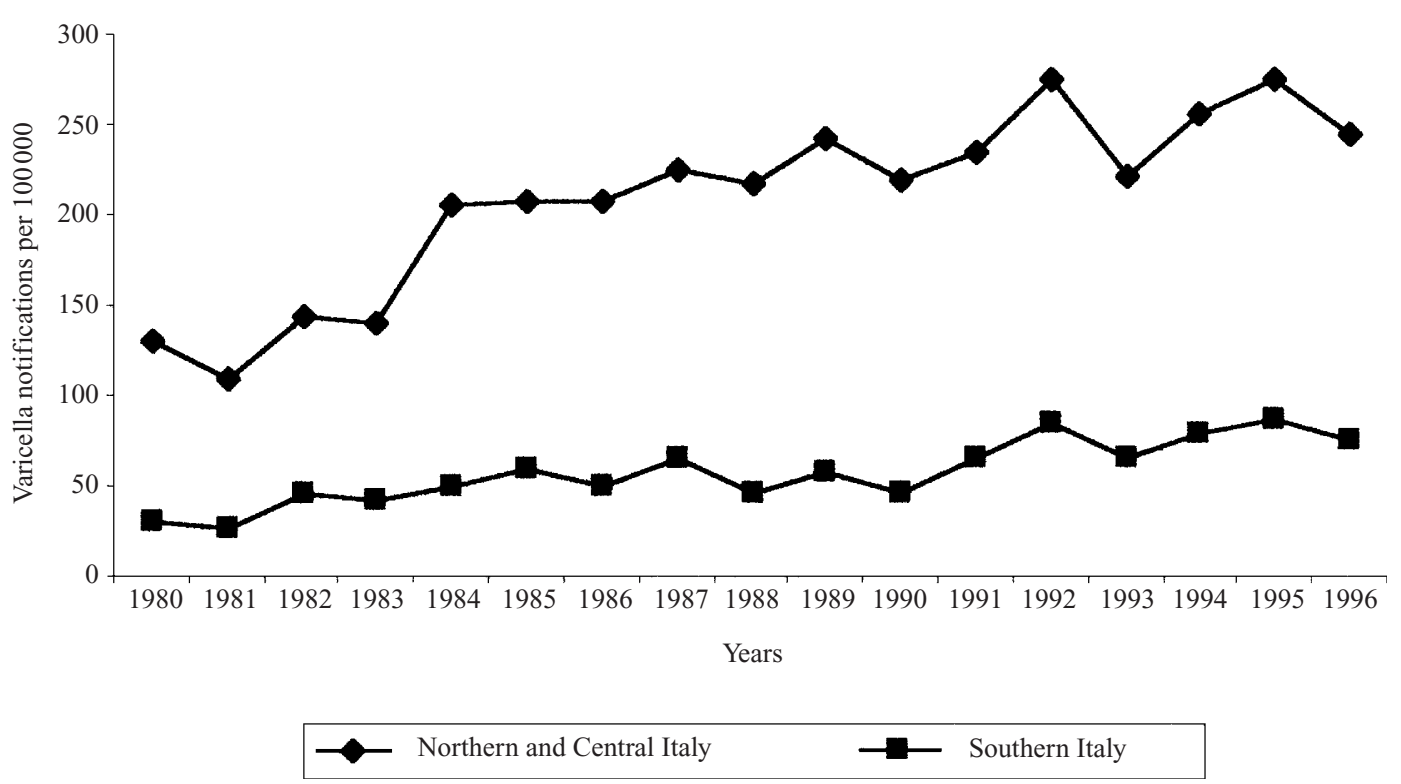

Fig. 4. Varicella morbidity by geographical area in Italy 1961-96.

$(P<0 \cdot 01) ; 25-44$ years $(P<0 \cdot 01)$; and $\geqslant 45$ years $(P<0.01)$ ] (Fig. 2). Specifically, the morbidity for the $0-4$ years age-group progressively increased in the various periods considered, whereas that for the 5-9 years age-group decreased in the period 1971-80 and significantly increased in the following periods. In the age-groups of 10-14, 25-44 and $\geqslant 45$ years, there was a progressive increase in morbidity, whereas in the 15-24 years age-group, there was a decrease in the period 1991-6, though the morbidity in this period remained significantly higher with respect to the periods $1961-70$ and $1971-80$.
When combining the data into two age-groups (i.e. $0-14$ years and $\geqslant 15$ years), the morbidity per 100000 inhabitants in the $0-14$ years age-group was 303.9 in $1961-70,271.9$ in $1971-80,533.8$ in 1981-90, and 925.6 in $1991-6(P<0 \cdot 01)$. The morbidity per 100000 inhabitants in the $\geqslant 15$ years age-group was 6.3 in 1961-70, 10.5 in 1971-80, 40.2 in 1981-90, and 38.9 in $1991-6(P<0 \cdot 01)$. Beginning in the 1970s, there was an increase in the percentage of varicella cases notified in the $\geqslant 15$ years age-group [i.e. $6.0 \%$ in $1961-70$, $10.5 \%$ in $1971-80,21 \cdot 1 \%$ in $1981-90$, and $17 \cdot 7 \%$ in 1991-6 $(P<0 \cdot 01)$ ] (Fig. 3). 


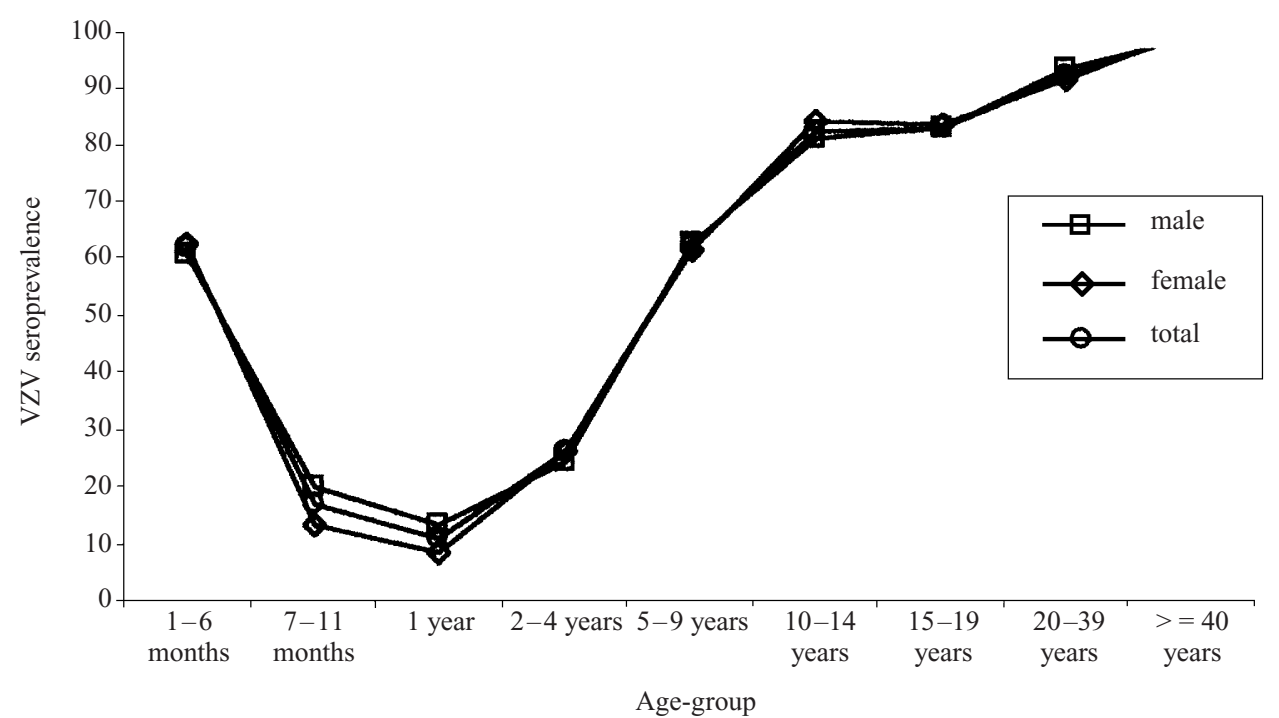

Fig. 5. Varicella-zoster virus seroprevalence by age-group and gender in Italy 1996-7.

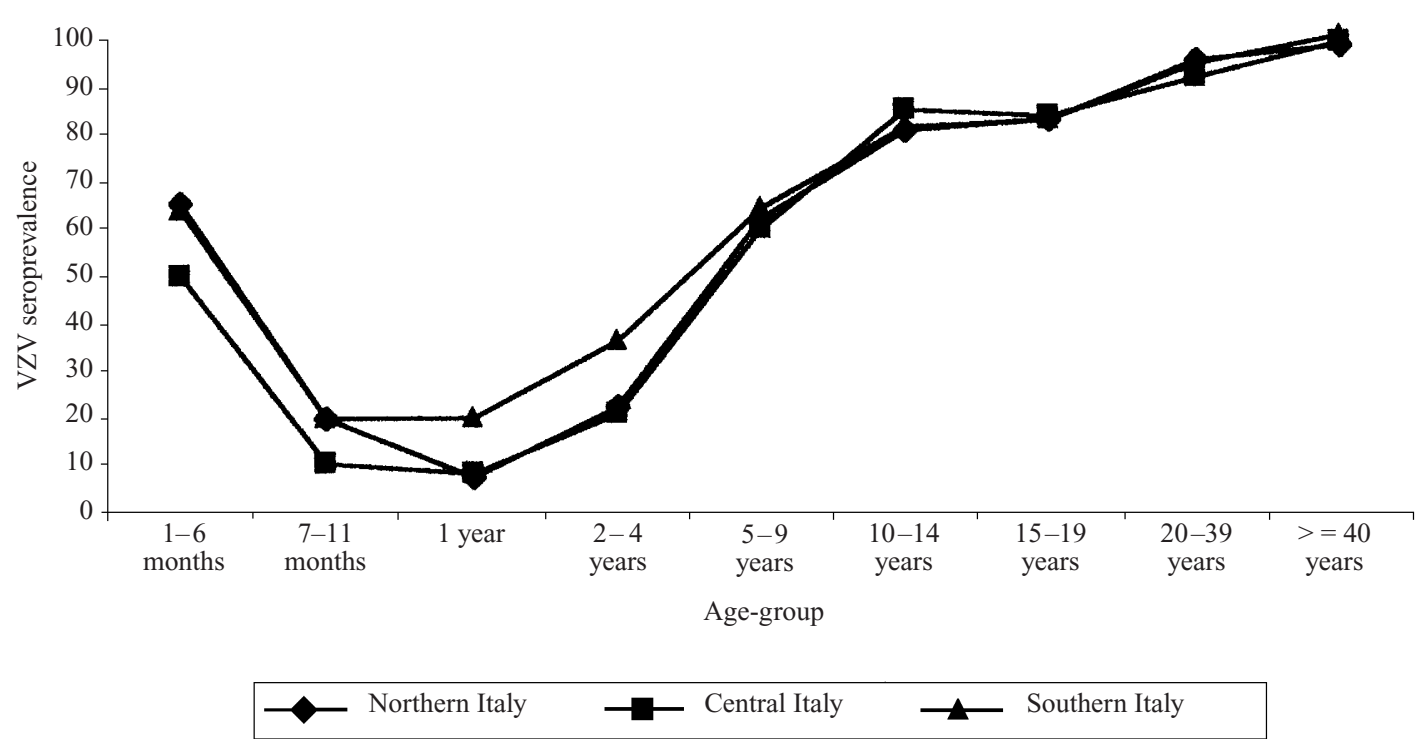

Fig. 6. Varicella-zoster virus seroprevalence by age-group and geographical area in Italy 1996-7.

The analysis of geographic trends in morbidity showed that although the temporal trend was practically identical, there was a significant difference in terms of the number of cases notified, and thus in morbidity, with a significantly lower number of notified cases in southern Italy, compared to northerncentral Italy (Fig. 4).

Of the 3569 serum samples collected from the 18 participating regions, 3179 were tested $; 1 \cdot 3 \%$ of these samples were borderline and were not considered in the data analysis. The national seroprevalence is shown in Figure 5: it progressively decreased among children in their first months of life, who were initially passively protected by the mother; it then began to increase following the first year of life and continued to increase, from $26.0 \%$ in the $2-4$ years age-group to $61.8 \%$ in the $5-9$ years age-group, reaching $82.1 \%$ in the 10-14 years age-group. The seroprevalence was identical by gender for all age-groups.

When considering the regions by age-group, the statistical analysis did not reveal any significant differences, except in the 2-4 years age-group $(P<0 \cdot 05)$. When dividing Italy into three geographic areas (i.e. northern, central, and southern Italy) (Fig. 6), the trend of seroprevalence was similar among the three; however, the percentage of persons with specific anti-VZV antibodies tended to increase earlier in southern Italy, with respect to the other areas. In fact, 


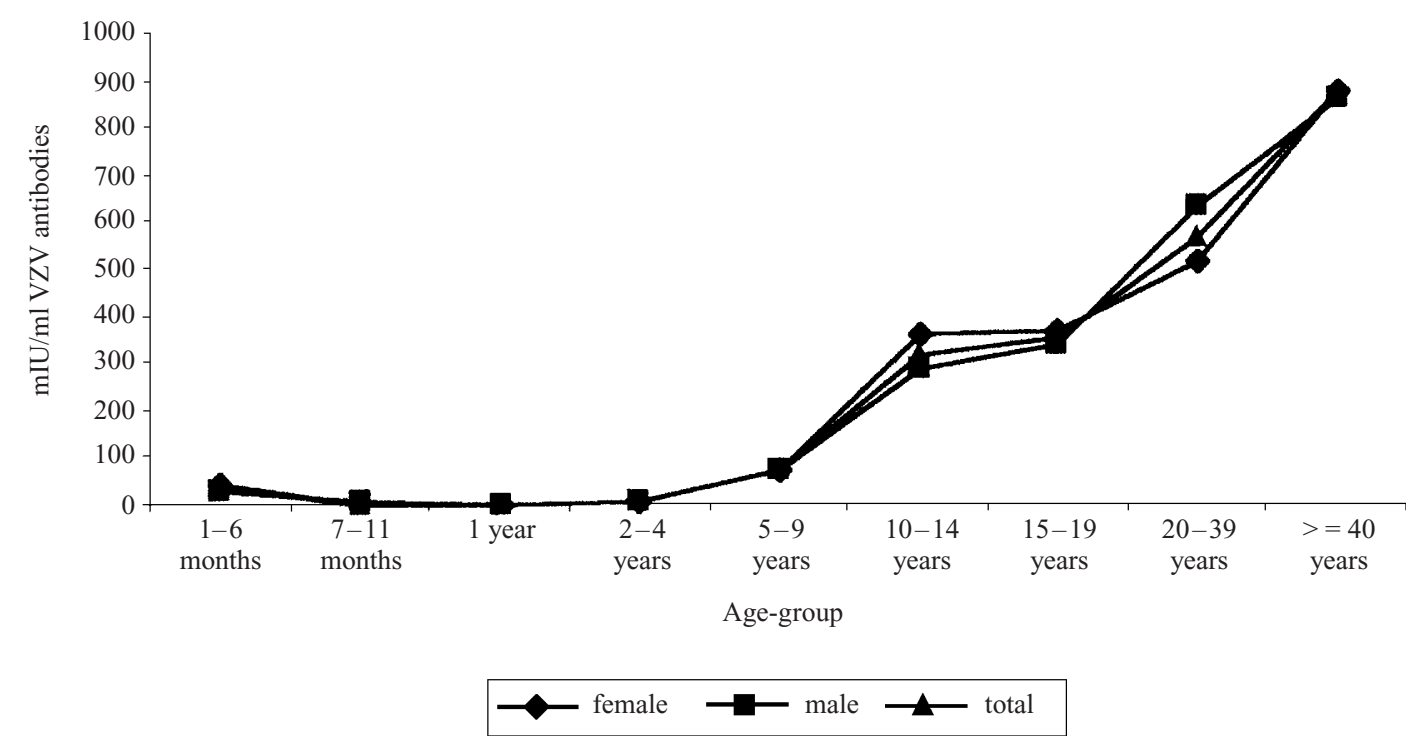

Fig. 7. Geometric mean titre of varicella-zoster virus antibodies by age-group and gender in Italy 1996-7.

the seroprevalence in the 2-4 years age-group was significantly greater in southern Italy, with respect to northern and central Italy $(P<0 \cdot 05)$.

These results are indirectly confirmed by the analysis of trends in GMT in the various age-groups (Fig. 7); the GMT began to increase in the $2-4$ years age-group, with an abrupt increase in the 5-9 years age-group. This trend of increase continued in all agegroups, indicating a persistent circulation of VZV in the population. No significant differences by gender were observed in the GMT.

\section{DISCUSSION}

In designing this seroprevalence study, we attempted to ensure that the sample was representative, following indications provided by the European Project for the Sero-epidemiological Surveillance of Vaccine-Preventable Diseases [20]. Although the samples from very young participants could have been collected from children with health problems (i.e. problems that would lead to varicella vaccination being recommended), the inclusion and exclusion criteria used in this study should have resulted in these children being excluded, thus avoiding an overestimation of seroprevalence.

The results of the present study confirm that in Italy, varicella typically involves children. In fact, the seroprevalence markedly increased from $25 \%$ among the 2-4 years age-group to $61 \cdot 8 \%$ among the 5-9 years age group $(P<0 \cdot 01)$. Nonetheless, there was a significant increase $(P<0.01)$ from the $5-9$ years agegroup to the 10-14 years group (61.8 and $82.1 \%$, respectively), whereas the seroprevalence remained stable among older individuals. These findings indicate that there is extensive circulation of VZV in Italy even among those age-groups not considered to be the most exposed, as already observed in other countries [21]; thus there is the possibility that the mean age of infection is increasing. Only age-specific time trends can demonstrate a shift in the age at infection.

This increase in age seems to be confirmed by the trend of varicella notifications: since the 1970s, there has been an increase in the percentage of cases reported among young adults. This could be due to the marked decrease in births, which has hindered the transmission of the virus from one susceptible individual to another. Nonetheless, there seems to have been a trend of progressive increase over time in varicella morbidity (Fig. 1). However, this increase can probably be attributed to a greater tendency of health care workers to notify cases, although it is known that for all communicable diseases, and in particular for childhood exanthems, there is still a great degree of undernotification. The notification data presented in this paper may not be an absolutely accurate record of events, since there is acknowledged to be variation of notification practice in different parts of Italy and the trend over time may not be accurate.

Taking these considerations in account, the increase in the percentage of cases notified among young adults may be a cause for alarm since the clinical course of varicella is generally more serious in adults and the incidence of complications is higher. Among 
specific population groups [e.g. among individuals with chronic renal failure or lymphoproliferative diseases; liver, bone-marrow and kidney transplant recipients; and immunocompromised individuals in general] varicella can be most severe or fatal [22-24]. The percentage of females of reproductive age who do not have specific anti-VZV antibodies (17.2\% among the 15-19 years age-group and $9 \cdot 2 \%$ among the 20-39 years age-group) indicates the risk of having pregnant women susceptible to VZV and confirms the abovereported epidemiological trend.

Of note is the different seroepidemiological trend found in the diverse geographic areas of Italy, which seems to indicate a greater circulation of VZV in southern Italy, compared to the rest of the country. However, this finding apparently contradicts the notification data, which show that morbidity is significantly higher in northern-central Italy. A possible explanation could be the lower age of acquisition in southern Italy and a particularly high degree of undernotification. It is also probable that in the regions of northern-central Italy, the spread of the virus has been facilitated by the fact that a greater percentage of the population lives in large urban and industrial areas.

These findings lead to several conclusions. First of all, the mandatory notification of communicable diseases in Italy must be improved. In fact, Italy's epidemiological surveillance system, like those of many other countries [16], is known to suffer from a great degree of undernotification [16]. To confirm the trend of progressive increase in the age of acquisition observed in this study, it is crucial to have precise and reliable age- and gender-specific data. These data are also fundamental for evaluating the suitability of establishing mass varicella vaccination programmes, as proposed by several countries [16]. The impact that a mass vaccination programme among children $12-18$ months of age could have on the epidemiological trend of the infection must be carefully considered. In fact, programmes that fail to reach high levels of coverage could lead to an increase in the mean age of acquisition of the infection, which should be avoided for communicable diseases in general [25] and especially for varicella.

That varicella already seems to be following this trend [26] emphasizes the need to be even more prudent before planning interventions of this type, despite the fact that a safe and effective vaccine is available [27]. In particular, Italy's varicella notification data needs to be properly verified. In light of the information available, it would apparently be more productive to limit the use of varicella vaccine to those individuals at risk, as currently recommended in Italy [18], and to verify the epidemiological and costbenefit impact of eventual vaccine campaigns targeting, for example, susceptible women of reproductive age or health-care personnel [28].

\section{ACKNOWLEDGEMENTS}

This work was partly supported by a grant from SmithKline Beecham SpA, Italy. We are grateful to M. Kanieff for editorial assistance and to R. Cerruti and N. Nigro for technical assistance.

\section{REFERENCES}

1. Weller TH. Varicella-herpes zoster virus. In: AE Evans, RA Kaslow, eds. Viral infections of humans. Epidemiology and control, IVth edition New York: Plenum Medical Book Company, 1997: 865-92.

2. Hope-Simpson RE. The nature of herpes zoster: a longterm study and a new hypothesis. Proc R Soc Lond 1965; 58: 9-20.

3. Preblud SR. Age-specific risks of varicella complications. Pediatrics 1981; 68: 14-7.

4. Miller E, Vurdien J, Farrington P. Shift in age in chickenpox. Lancet 1993; i: 308-9.

5. Choo PW, Donahue JG, Manson JE, Platt R. The epidemiology of varicella and its complications. J Infect Dis 1995; 172: 706-12.

6. Enders G, Miller E, Cradock-Watson J, Bolley I, Ridehalgh M. Consequences of varicella and herpes zoster in pregnancy: prospective study of 1739 cases. Lancet $1994 ; 343$ : 1548-50.

7. Pastuszak AL, Levy M, Shick B, et al. Outcome after maternal varicella infection in the first 20 weeks of pregnancy. N Engl J Med 1994; 330: 901-5.

8. Meyers JD. Congenital varicella in term infants: risk reconsidered. J Infect Dis 1974; 129: 215-7.

9. Brunell PA. Varicella in pregnancy, the fetus and the newborn: problems in management. J Infect Dis 1992; 166 (Suppl. 1): S42-7.

10. Gershon AA, Takahashi M, White CJ. Varicella vaccine. In: Plotkin SA, Orenstein WA, eds. Vaccines, 3rd edn. W. B. Saunders Co. 1999; 475-507.

11. Weller TH. Varicella: historical perspective and clinical overview. J Infect Dis 1996; 174 (Suppl. 3): S306-9.

12. White E. Chickenpox in Kerala, India. Ind J Publ Hlth 1950; 22: 141-51.

13. Sinha DP. Chickenpox - a disease predominantly affecting adults in West Bengal, India. Int $\mathbf{J}$ Epidemiol 1976; 5: 367-74.

14. Takahashi M, Otsuka T, Okuno Y, Asano Y, Yakazi T, Isomura S. Live vaccine used to prevent the spread of varicella in children in hospital. Lancet 1974; ii: 1288-90. 
15. Brisson M, Edmunds WJ, Gay NJ, Law B, De Serres G. Analysis of varicella vaccine breakthrough rates: implications for the effectiveness of immunisation programs. Vaccine $2000 ; 18$ : 2775-8.

16. CDC. Prevention of varicella. Recommendations of the Advisory Committee on Immunization Practices (ACIP). MMWR 1996; 45: RR11, 1-36.

17. Holmes SJ. Review of recommendations of the Advisory Committee on Immunization Practices, Centers for Disease Control and Prevention, Varicella vaccine. J Infect Dis 1996; 174 (Suppl. 3): S342-4.

18. Ministero della Sanità. Circolare n. 8 del 10/03/1992: Indicazioni della vaccinazione antivaricella in categorie di soggetti a rischio.

19. Ministero della Sanità. Decreto Ministeriale 15 dicembre 1990. Sistema di sorveglianza sulle malattie infettive. Gazzetta Ufficiale 1991; 6: 8-18.

20. The development of a European sero-epidemiology collaboration for the investigation of vaccine preventable disease: the European Sero-Epidemiology Network. Biomed 1996-1999. Contract number: PL951039.

21. Fairley CK, Miller E. Varicella-zoster virus epidemi-
ology-A changing scene? J Infect Dis 1996; 174 (Suppl. 3): S314-9.

22. Preblud SR. Varicella: complications and costs. Pediatrics 1986; 78 (Suppl.): 728-35.

23. Feldman S, Hughes WT, Daniel CB. Varicella in children with cancer: seventy-seven cases. Pediatrics 1975; 56: 388-97.

24. Miller E, Marshall R, Vurdien J. Epidemiology, outcome and control of varicella-zoster infection. Rev Med Microbiol 1993; 4: 222-30.

25. Anderson RM, May RM. Immunization and herd immunity. In: A Lancet Review. Modern vaccines. Current practice and new approaches. E. Arnold, ed. 1990; 24-33.

26. Gray GC, Palinkas LA, Kelley PW. Increasing incidence of varicella hospitalizations in United States army and navy personnel: are today's teenagers more susceptible? Should recruits be vaccinated? Pediatrics $1990 ; 86: 867-73$

27. Asano Y. Varicella vaccine: the Japanese experience. J Infect Dis 1996; 174 (Suppl. 3): S310-3.

28. Gray AM, Fenn P, Weinberg J, Miller E, McGuire A. An economic analysis of varicella vaccination for health care workers. Epidemiol Infect 1997; 119: 209-20. 